\title{
Erkenntnisse vom ASCO 2008
}

\author{
Gesprächsleitung: Nadia Harbeck, München; Christoph Thomssen, Halle \\ Teilnehmer: $\quad$ Nicolai Maass, Kiel; Andreas Schneeweiss, Heidelberg; \\ Elmar Stickeler, Freiburg i. Br.; Barbara Schmalfeldt, München; \\ Eva Kantelhardt, Halle
}

Frage 1: Wie definieren Sie den Stellenwert der Aromatasehemmer in der adjuvanten Therapie der prä- oder perimenopausalen Patientin?

Maass: Die auf dem ASCO 2008 vorgestellte ABCSG-12-Studie von Gnant et al. zeigt erstmals eine potentielle Wirkung des Aromatasehemmers Anastrozol in der Prämenopause. Diese Daten sind jedoch präliminär und hoch selektiv. Zum jetzigen Zeitpunkt bleibt Tamoxifen Standard in der adjuvanten Therapie der prämenopausalen Patientin. Aromatasehemmer sollten nur im Rahmen von Studien (unter anderem SOFT- und TEXT-Studie) oder bei vorzeitiger Postmenopause (nach Adnektomie) Einsatz finden.

Schneeweiss: Aromatasehemmer in Kombination mit Goserelin sind in der Prämenopause nur bei eindeutigen Kontraindikationen gegenüber Tamoxifen eine alternative Option. Aber Vorsicht, da Goserelin nicht bei allen Patientinnen eine Ovarialinsuffizienz induziert, kann gegebenenfalls Bestimmung von E2 notwendig sein.

Stickeler: Mit den auf dem ASCO publizierten Daten der ABCSG-12-Studie sehe ich den ersten soliden Nachweis erbracht, dass diese Kombinationstherapie bei prä- oder perimenopausalen Patientinnen eingesetzt werden kann. Insbesondere bei Kontraindikationen gegen Tamoxifen sehe ich hier eine Indikation. Standard bleibt für mich in dieser Situation weiterhin Tamoxifen.

Schmalfeldt: Bisher gibt es noch zu wenige Daten im Hinblick auf die Auswirkung auf die Östrogenspiegel; der Einsatz von Aromatasehemmern in der Prämenopause sollte nur mit GnRH-(Gonadotropin Releasing Hormon-)Analoga erfolgen.

Kantelhardt: Da Aromatasehemmer bei prä- bzw. perimenopausalen Patientinnen keine ausreichende Unterdrückung der ovariellen Funktion bieten, sollten sie nur im Einzelfall, bei Kontraindikationen gegen Tamoxifen (z.B. Thrombose) und in Kombination mit GnRH-Analoga gegeben werden. Die aktuellen Daten der ABCSG-12-Studie zeigten im Vergleich der Aromatasehemmer mit Tamoxifen über 3 Jahre bei 60 Monaten Follow-up keinen Vorteil. Die Ergebnisse der SOFT- und TEXT-Studie mit ähnlicher Fragestellung stehen aus.

\section{Frage 2: Welchen Stellenwert haben die Antrazykline in der adjuvanten Chemotherapie?}

Maass: Die Antrazykline sind unverändert Standard in der adjuvanten Therapie, und zwar vorwiegend die Dreierkombination. Bei Kontraindikationen, insbesondere bei kardialen Risiken, erwägen wir Taxan/Cyclophosphamid (TC) als Alternative.

Schneeweiss: Anthrazykline bleiben weiterhin Standard, da alle Studien zu prädiktiven Faktoren bisher retrospektiv oder noch nicht abschließend ausgewertet sind. In Zukunft könnten allerdings Anthrazyklin-freie Protokolle die Anthrazykline bei vielen Patientinnen ersetzen, wenn reife prospektive Studien die Gleichwertigkeit oder Überlegenheit bei gleicher oder geringerer Langzeittoxizität bewiesen haben.

Stickeler: Die Anthrazykline haben in der Adjuvanz weiterhin einen festen Stellenwert, wenn auch die molekularbiologischen Unterschiede einzelner Tumorentitäten (Topo-IIKoamplifikation) und das daraus resultierende unterschiedliche Ansprechen auf einzelne Substanzen immer mehr an Bedeutung gewinnen und die Therapien zielgenauer ausrichtet werden.

Schmalfeldt: Aufgrund der hohen Evidenz sind Anthrazykline die Medikamente der ersten Wahl. Eine kritische Nutzen-Risi-

\begin{tabular}{ll}
\hline KARGER & ( 2008 S. Karger GmbH, Freiburg \\
Fax +49 7614520714 & Accessible online at: \\
$\begin{array}{l}\text { E-mail Information@Karger.de } \\
\text { www.karger.com }\end{array}$ & www.karger.com/brc
\end{tabular}


ko-Abwägung ist jedoch nötig, da inzwischen für Taxan-haltige Anthrazyklin-freie Schemata Daten aus Phase III in der Adjuvanz vorliegen.

Kantelhardt: Anthrazykline sind weiterhin das wichtigste Standbein der adjuvanten Chemotherapie. Bei Kontraindikation wegen Begleiterkrankung der Patientin können Anthrazyklin-freie Regime erwogen werden (Doxorubicin/Carboplatin/Trastuzumab oder Doxorubicin/Cyclophosphamid). Möglicherweise können prädiktive Faktoren in Bezug auf das Ansprechen benutzt werden: An 121 Patientinnen mit ERnegativem Mammakarzinom und neoadjuvanter Epirubicingabe konnte die Amplifikation des Topoisomerase-Gens (in $31 \%$ der ausschließlich HER2 überexprimierenden Fälle) die pathologische Komplettremission signifikant vorhersagen; mRNA und Protein zeigten keine Korrelation, so Desmedt et al. auf dem ASCO 2008.

\section{Frage 3: Welchen Patientinnen empfehlen Sie eine adjuvante Bisphosphonat-Therapie?}

Maass: Trotz einer Reihe von Studien (auch hier: ABCSG-12Studie) empfehlen wir eine adjuvante Bisphosphonat-Therapie, entsprechend der AGO-Empfehlungen, nur im Rahmen von Studien, z.B. der Natan-Studie. In Kombination mit Aromatasehemmern in der adjuvanten Therapie empfehlen wir die zusätzliche Gabe von Bisphosphonaten bei Risikopatienten bzw. pathologischem Dexa-Scan.

Schneeweiss: Bisher empfehle ich diese Therapie nur prä- oder perimenopausalen Patientinnen, die adjuvant eine reine endokrine Therapie bekommen, sowie in Einzelfällen, z.B. bei Tumoreinzelzellnachweis im Knochenmark durch ein standardisiertes Verfahren.

Stickeler: Auf der Basis der aktuellen Datenlage, welche neben den beschriebenen Therapieerfolgen der Bisphosphonate in der Adjuvanz auch die Nebenwirkungen (und Kosten) berücksichtigen sollte, würde ich der jungen Risikopatientin die adjuvante Bisphosphonat-Therapie empfehlen.

Schmalfeldt: Prämenopausalen Patientinnen mit alleiniger endokriner Therapie sowie allen Hochrisiko-Patientinnen.

Kantelhardt: Die 2×2-faktoriell gestaltete ABCSG-12-Studie von Gnant et al. zeigte bei 1800 operierten, Hormonrezeptorpositiven, prämenopausalen Patientinnen mit weniger als 11 befallenen Lymphknoten, dass die Hinzunahme von 4 mg Zoledronat alle 6 Monate zur Therapie mit Goserelin und Tamoxifen oder Anastrozol bei einem medianen Follow-up von 60 Monaten das krankheitsfreie Überleben (einschließlich aller Todesfälle) um 36\% und das rezidivfreie Überleben um 35\% jeweils signifikant erhöhen kann. Für den breiten Einsatz in der Adjuvanz sollten wegen der bisher widersprüchlichen Ergebnisse weiterer Studien $(2 \times$ negative, $1 \times$ positive Wirkung) die Ergebnisse der noch ausstehenden Studien (z.B. NATAN, GAIN, NSABP B-34, AZURE) abgewartet werden. Bei triple-negativen Patientinnen könnte man die Ergebnisse extrapolieren und Bisphosphonate mangels anderer Therapieoptionen erwägen.

\section{Frage 4: Wie unterscheiden Sie «basal-like» und triple-negative Tumoren in der klinischen Routine und wie behandeln Sie diese Tumoren in der adjuvanten und in der metastasierten Situation?}

Maass: Die triple-negativen Tumoren gelten als prognostisch ungünstig, insbesondere aufgrund mangelnder therapeutischer Möglichkeiten. Eine Chemotherapie wird sowohl adjuvant als auch metastasiert allgemein indiziert. Anthrazykline und Taxane werden hier von uns nach Standard eingesetzt. Eine klinische Unterscheidung zwischen triple-negativen und basallike Tumoren erfolgt von uns bislang nicht. Eine Therapie erfolgt, wenn möglich, im Rahmen der Beatrice-Studie.

Schneeweiss: Nicht eine Negativselektion sollte erfolgen, sondern eine Positivselektion anhand zusätzlicher immunhistochemischer Marker wie CK5/6 und CK14, gegebenenfalls EGFR und CD117 (c-kit). Momentan behandeln wir diese Karzinome noch wie andere High-risk-Karzinome, da Daten aus prospektiven Studien zur besonderen Sensitivität gegenüber bestimmten Substanzen (Platinsalze, Anti-EGFR etc.) fehlen.

Stickeler: In der klinischen Routine unterscheiden wir «basallike» und triple-negative Tumoren nicht! Wir behandeln sie in Abhängigkeit vom Alter der Patientin mit Taxanen und/oder Platin-haltigen Therapien in der Adjuvanz und bei Metastasierung mit Taxan + Bevacizumab oder Taxanen.

Schmalfeldt: Ich empfehle eine Therapie entsprechend den klassischen klinischen Prognosefaktoren.

Kantelhardt: Derzeit gibt es weder für die «basal-like» noch für die $15 \%$ triple-negativen Tumoren besondere Therapieempfehlungen in der klinischen Routine - daher ist eine genaue Identifizierung der «basal-like» Tumoren außerhalb von Studien nicht erforderlich. Bei bekannter schlechter Prognose der triple-negativen Fälle sollte Wert auf eine intensive Chemotherapie gelegt werden, besonders die neoadjuvante Therapie im Rahmen der GeparQuinto-Studie bietet gute Möglichkeiten; oder adjuvant eine Anlehnung an den Standardarm der GAIN-Studie (dosisintensiviert, intervallverkürzt Epirubicin, Paclitaxel und Cyclophosphamid). Eine routinemäßige Gabe Platin-haltiger Chemotherapien sollte erst nach den Ergebnissen der entsprechenden Studien erfolgen. In der meta- 
stasierten Situation können firstline Paclitaxel und Bevacizumab zur Anwendung kommen, bei den Monotherapien stehen gerade auch Platin-Derivate zur Verfügung.

\section{Frage 5: Ist für Sie die alleinige Kombination zweier oder mehrerer zielgerichteter Therapien eine klinische Therapieoption?}

Maass: Auf dem ASCO 2008 erscheinen zunehmend Studien zur Kombination zweier Targeted-Therapien. Ein Verzicht auf eine Chemo- bzw. endokrine Therapie erfolgt nur im äußersten Einzelfall bzw. innerhalb von Studien. Ich erwarte jedoch eine zunehmende Indikation zur Kombination mehrerer zielgerichteter Therapien.

Schneeweiss: Sobald Daten aus prospektiven randomisierten Studien vorliegen, ist für mich die alleinige Kombination zweier oder mehrerer zielgerichteter Therapien bei entsprechend selektionierten Patientinnen auf jeden Fall eine klinische Therapieoption.

Stickeler: Auf des Basis der bisher publizierten Daten nicht.

Schmalfeldt: Nein, aufgrund des unklaren additiven und potenzierten Nebenwirkungsrisikos nicht.

Kantelhardt: Dies ist sicher derzeit eine Option im Rahmen von Studien wie der GeparQuinto-Studie (www.germanbreast group.de). Interessante präklinische Konzepte sind verfügbar. Für die klinische Routine fehlen bisher Daten.

\section{Frage 6: Wo sehen Sie derzeit den Stellenwert der Antiangiogenese-Therapie?}

Maass: Die antiangiogenetische Therapie, überwiegend basierend auf einer VEGF-Hemmung, ist Teil der zunehmend zielgerichteteren Therapieoptionen. Eine Reihe von Angiogenesehemmern befindet sich in der klinischen Überprüfung. Ihr Stellenwert wird weiter zunehmen. $\mathrm{Zu}$ den klinisch relevantesten Vertretern der Angiogenesehemmer beim Mammakarzinom zählt zum jetzigen Zeitpunkt das Bevacizumab.

Schneeweiss: Als Firstline-Therapie in Kombination mit einem Taxan bei Patientinnen mit HER2-negativem, metastasierten Mammakarzinom, bei denen rasch eine Remission erzielt werden muss - also als alternative Option mit differentem Nebenwirkungsprofil zu einer Taxan-haltigen Firstline-Kombinationschemotherapie.

Stickeler: Die Antiangiogenese-Therapie ist für mich ein interessantes Konzept für Studien in der Adjuvanz (BETH) oder neoadjuvant (GeparQuinto). Ich halte sie für etabliert in der metastasierten Situation.

Schmalfeldt: Entsprechend der Zulassung als Firstline-Therapie in Kombination mit Paclitaxel

Kantelhardt: Nach Miles et al. kann Bevacizumab (7,5 oder $15 \mathrm{mg} / \mathrm{kg}$ alle 3 Wochen) zusätzlich zu Docetaxel bei Patientinnen mit HER2-negativem, metastasierten Mammakarzinom firstline mit akzeptabler Toxizität ein längeres progressionsfreies Überleben (HR 0,79 bzw. 0,72) sowie ein besseres Ansprechen (55 bzw. 63\% gegenüber Docetaxel alleine) gewährleisten. Diese Strategie sollte in der Routine angewendet werden. Auch zu Sunitinib und Pazopanib, d.h. zu Antiangionese-Wirkstoffen auf der Ebene der Rezeptortyrosinkinasen existieren bereits interessante Studiendaten.

\section{Frage 7: Haben sich die Standards der adjuvanten Chemotherapie nach dem ASCO geändert?}

Maass: Grundlegend haben sich die Standards in der adjuvanten Chemotherapie seit dem ASCO nicht geändert. Die Bedeutung der Taxane und von Trastuzumab ist weiter bestätigt worden.

Schneeweiss: Die Standards der adjuvanten Chemotherapie haben sich nicht geändert, aber die Grenzen wurden aufgeweicht. So diskutiere ich jetzt schon bei jeder nodal-negativen High-risk-Situation (z.B. beim triple-negativen Mammakarzinom) den Einsatz von TAC (Docetaxel, Doxorubicin, Cyclophosphamid).

Stickeler: Aktuell für mich in der Standardsituation nicht!

Schmalfeldt: Nein.

Kantelhardt: Die Standards sind weiterhin dieselben. Weitere Optionen für spezielle Patientinnen können durch neue Daten erwogen werden (z.B. Bisphosphonate in der adjuvanten Situation). Die Umsetzung prädiktiver Faktoren in die Routine wird gerade bei der zunehmenden Vielzahl der Substanzen und Strategien dringend erwartet: Welche Hormontherapie, welche Chemotherapie und welche zielgerichteten Therapien sind bei der je individuellen Patientin unter Erwartung welcher Nebenwirkungen sinnvoll?

\section{Frage 8: Was hat Sie auf dem ASCO besonders beeindruckt?}

Maass: Die Zunahme einer Reihe von Studien zur sogenannten zielgerichteten Therapie. Insbesondere in der metastasierten Situation werden vorwiegend klassische Chemotherapeu- 
tika oder neue Targeted-Therapien vorgestellt. Auch die chemotherapiefreien Kombinationstherapien mehrerer «small molecules» befinden sich im Vormarsch.

Schneeweiss: Die Professionalität der Organisation, die Größe des Kongresses und die zumeist hohe Qualität der Vorträge, insbesondere der Übersichtreferate, die den Besucher erahnen lassen, wohin sich die Onkologie entwickelt.

Kantelhardt: Die Bisphosphonat-Diskussion ist sehr spannend - gerade mit den Überlegungen zur Metastasierung («seed and soil»-Konzept). Die Überlegungen zur Therapie des «basal-like» Karzinoms zeigen neue Perspektiven auf. Vielleicht kann man durch besseres Verständnis der Tumorbiologie neue Therapieoptionen finden.

\section{Teilnehmer}

Prof. Dr. med. Nicolai Maass

Klinik für Gynäkologie und Geburtshilfe

Universitätsklinikum Schleswig-Holstein, Campus Kiel

Michaelisstr. 161, 24105 Kiel, Deutschland

Tel. +49 431 597-2100/2101, Fax -1247

nmaass@email.uni-kiel.de

Prof. Dr. med. Andreas Schneeweiss

Sektionsleiter Onkologische Ambulanz und Tagesklinik

der Universitäts-Frauenklinik

Voss-Str. 9, 69115 Heidelberg, Deutschland

Tel. +49 6221 56-7856, Fax -7920

andreas.schneeweiss@med.uni-heidelberg.de

Prof. Dr. med. Elmar Stickeler

Leitender Oberarzt Comprehensive Cancer Center Freiburg

Universitätsfrauenklinik Freiburg

Hugstetter Str. 55, 79106 Freiburg, Deutschland

Tel +49 761 270-3148, Fax -3148

elmar.stickeler@uniklinik-freiburg.de

Univ.-Prof. Dr. med. Barbara Schmalfeldt

Leitende Oberärztin Frauenklinik

Klinikum rechts der Isar der TU München

Ismaninger Str. 22, 81675 München, Deutschland

Tel. +49 89 4140-5414/2433 oder 2420, Fax -4846

barbara.schmalfeldt@lrz.tum.de

Dr. med. Eva Kantelhardt

Universitätsfrauenklinik

Ernst-Grube-Str. 40, 06120 Halle, Deutschland

Tel. +49 345 557-1847, Fax -1504

eva.kantelhardt@medizin.uni-halle.de 